\title{
Understanding Comorbidity Profiles and Their Effect on Treatment and Survival in Patients With Colorectal Cancer
}

\author{
Erin E. Hahn, PhD, MPHa; Michael K. Gould, MD, MSª Corrine E. Munoz-Plaza, MPHª; Janet S. Lee, MSª \\ Carla Parry, $\mathrm{PhD}, \mathrm{MSW}, \mathrm{MA}^{\mathrm{b}}$; and Ernest Shen, $\mathrm{PhD}^{\mathrm{a}}$
}

\begin{abstract}
Background: Patients with colorectal cancer (CRC) commonly present at an older age with multiple comorbid conditions and complex care needs at the time of diagnosis. Clusters of comorbid conditions, or profiles, have not been systematically identified in this patient population. This study aimed to identify clinically distinct comorbidity profiles in a large sample of patients with CRC from an integrated healthcare system, and to examine the effect of comorbidity profiles on treatment and survival. Methods: In this retrospective cohort study, we used latent class analysis (LCA) to identify comorbidity profiles in a sample of 7,803 patients with CRC diagnosed between 2008 and 2013. We identified treatment received from electronic health records and used Cox proportional hazards analysis to examine the effect of comorbidity class on survival. We also examined the effect of comorbidity profile on receipt of guideline-recommended treatment. Results: Median age at diagnosis was 66 years, $52 \%$ of patients were male, and $48 \%$ were nonwhite. A plurality had stage $0-I$ disease (42\%), with $22 \%$ stage II, $22 \%$ stage III, and $14 \%$ stage IV. More than half ( $59 \%)$ had $\leq 1$ comorbid condition, whereas $19 \%$ had $\geq 4$ comorbidities. LCA identified 4 distinct comorbidity classes. Classes were distinguished by the presence or absence of vascular and/or respiratory disease and diabetes with complications, as well as progressively greater Charlson comorbidity index scores. Comorbidity class was independently associated with treatment selection $(P<.001)$ and survival $(P<.001)$. Conclusions: Patients with $C R C$ can be described by 4 distinct comorbidity profiles that are independent predictors of treatment and survival. These results provide insight into how comorbidities cluster within patients with CRC. This work represents a shift away from simple counting of comorbid conditions and toward a more nuanced understanding of how comorbidities cluster within groups of patients with CRC.
\end{abstract}

J Natl Compr Canc Netw 2018;16(1):23-34 doi: 10.6004 /jnccn.2017.7026

Colorectal cancer (CRC) is the third most common cancer diagnosed in the United States. ${ }^{1}$ Approximately 140,000 new CRC cases were diagnosed in 2016, and there are currently more than 1 million survivors. ${ }^{1}$ These numbers will continue to increase due to advances in cancer detection and treatment, as well as the aging of the population. ${ }^{2,3}$ This increasing patient population, with a mean age at diagnosis of 69 years, frequently

From the aDepartment of Research \& Evaluation, Kaiser Permanente Southern California, Pasadena, California; and 'batient Centered Outcomes Research Institute (PCORI), Washington, DC.

Submitted April 13, 2017; accepted for publication August 17, 2017.

The authors have disclosed that they have no financial interests,

arrangements, affiliations, or commercial interests with the manufacturers of any products discussed in this article or their competitors. presents with multiple comorbid conditions and complex care needs at the time of diagnosis. A recent study found that almost half of patients with colon cancer had $\geq 3$ comorbidities, and patients with co-occurring diabetes spent more resources on medical treatment than any other group, excluding select patients with lung cancer. ${ }^{4}$ Additionally, evidence suggests older patients and those with a higher burden of comorbidity are less likely to re-

Author contributions: Study concept and design: Hahn, Gould, Parry. Data acquisition: Hahn, Gould, Lee, Shen. Data analysis and interpretation: Hahn, Gould, Munoz-Plaza, Lee, Parry, Shen. Manuscript preparation: Hahn, Munoz-Plaza. Critical revision: Hahn, Gould, Lee, Parry, Shen. Final approval of manuscript: Hahn, Gould, Munoz-Plaza, Lee, Parry, Shen. Correspondence: Erin E. Hahn, PhD, MPH, Department of Research and Evaluation, Kaiser Permanente Southern California, 100 South Los Robles Avenue, 2nd Floor, Pasadena, CA 91101. E-mail: Erin.E.Hahn@kp.org 
ceive and complete standard cancer treatments (eg, surgery, chemotherapy, radiation) and have lower rates of overall survival (OS). ${ }^{5-12}$

Effectively managing CRC treatment in patients with comorbid conditions is an important clinical issue. Determining how comorbid conditions cluster together, impact treatment selection, and ultimately influence prognosis in CRC is a critically understudied area. Studies have documented comorbidity index scores or the impact of a single comorbid condition (eg, diabetes) on treatment and survival for patients with $\mathrm{CRC}^{13-15}$; however, few if any have examined the impact of clusters of co-occurring comorbid conditions on treatment and OS. The objectives of this study were to identify clinically distinct comorbidity subgroups in patients with CRC from a large integrated healthcare system and to examine the effect of these subgroups on treatment selection and OS, addressing our a priori hypothesis that existing structured data can be used to determine if and how certain comorbid conditions cluster among patients with CRC. In addition, we examined the relationship between comorbidity clusters, treatment selection, and survival.

\section{Methods}

\section{Setting}

We performed a retrospective, observational cohort study using longitudinal data from Kaiser Permanente Southern California (KPSC), a large integrated healthcare system providing care to more than 4.2 million members. KPSC clinicians provide care at 14 medical centers across the region, and have a comprehensive Epic-based electronic health record (EHR) system, implemented in 2007. KPSC membership is ethnically and economically diverse, and is similar to the general population of Southern California. ${ }^{16}$ This study was approved by the KPSC Institutional Review Board (IRB\# 10458).

\section{Patients}

We identified patients diagnosed with first CRC occurrence (ICD-9 codes 153-154.1) between January 1, 2008, and December 31, 2013, from the KPSC cancer registry, a SEER-affiliated registry. We restricted the sample to patients aged $\geq 18$ years at diagnosis; those diagnosed between the ages of 18 and 64 years were included in order to include the comorbidity profiles of younger patients and to explore the potential influence of comorbidity on their treatment and survival, given that most existing studies focus on those diagnosed at age $>65$ years. We excluded patients with missing or unknown cancer stage, duplicate records, and nonanalytic cases (Figure 1). Nonanalytic cases are defined as patients who did not receive their cancer diagnosis and first course of treatment at KPSC. Patients who had KPSC membership but zero recorded physician visits before the date of CRC diagnosis were also excluded.

\section{Data Sources and Variables}

Variables from the KPSC tumor registry data included age at diagnosis, stage of disease (AJCC stage), histology, and treatment types. Available treatment data included receipt of chemotherapy (yes/ no), receipt of radiation (yes/no), and surgical type, in accordance with SEER reporting standards. Detailed treatment data, such as adjuvant/neoadjuvant chemotherapy, were not available. The KPSC EHR provided sociodemographic variables including date of birth, sex, race/ethnicity, body mass index (BMI), marital status, insurance type, and education and income (geo-coded at the census block or block group when available). Comorbid conditions were captured from the EHR by using the Deyo adaptation of the Charlson comorbidity index (DaCCI), which provides a standardized list of ICD diagnostic codes (ICD-9) that define each comorbid condition. We included ICD-9 codes that appeared at least once up to 4 years before the $\mathrm{CRC}$ diagnosis ${ }^{17-19}$ to capture comorbid conditions with high sensitivity. We selected the DaCCI for its broad generalizability and ability to be replicated in multiple settings, because the required data can be captured by administrative claims data and/or EHRs. We included the following chronic conditions: myocardial infarction, congestive heart failure, peripheral vascular disease, cerebrovascular disease, dementia, chronic obstructive pulmonary disease, connective tissue disease, rheumatic disease, peptic ulcer disease, mild liver disease, diabetes with and without complications, paraplegia/ hemiplegia, renal disease, cancer (excluding the index CRC cancer), moderate or severe liver disease, metastatic carcinoma, and AIDS/HIV. KPSC membership files and death data sets, obtained from the California State Death Master Files and the Social 
Colorectal Cancer Comorbidity Profiles

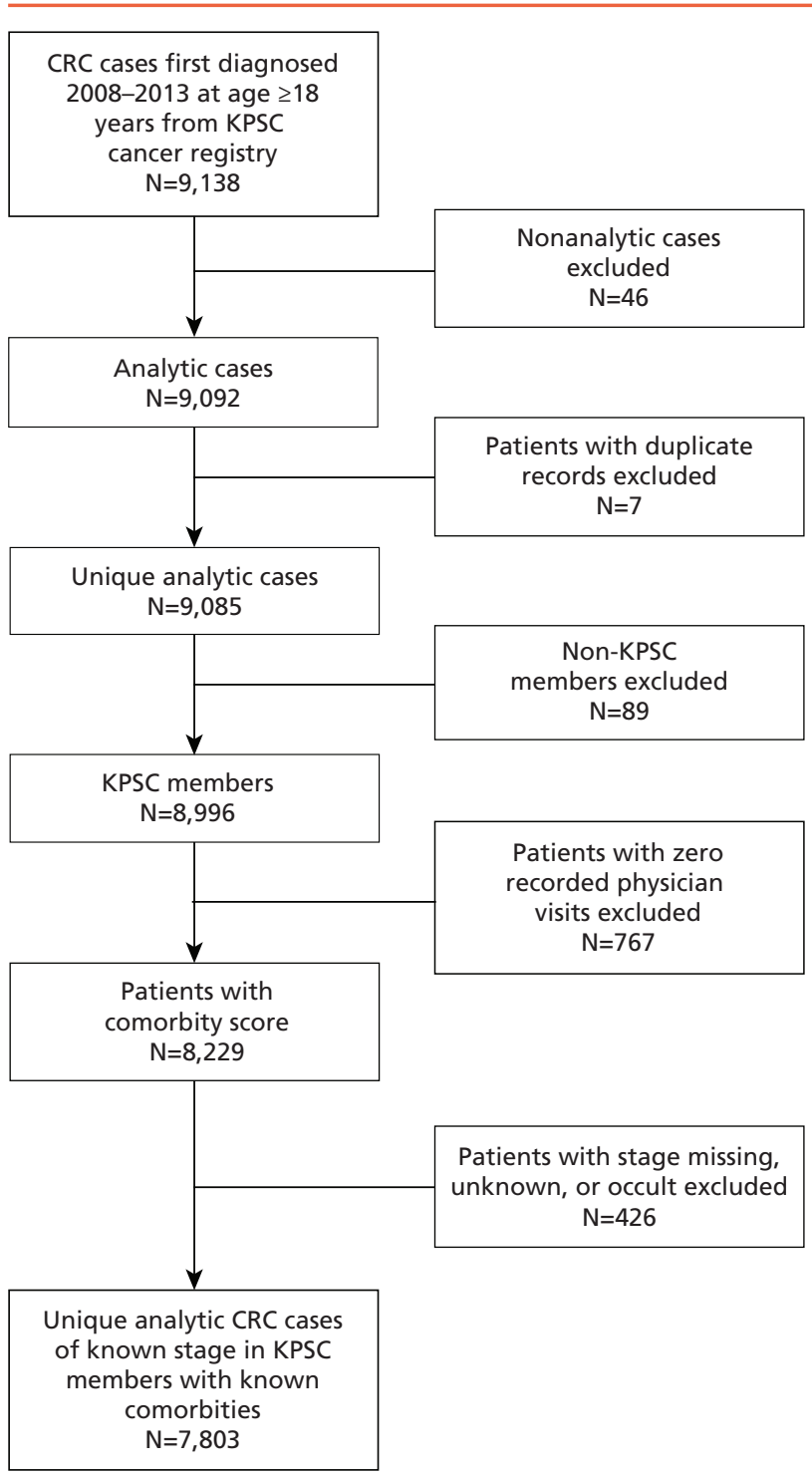

Figure 1. Cohort diagram for patients diagnosed with first colorectal cancer (CRC) between 2008-2013 within Kaiser Permanente Southern California (KPSC).

Security Administration Death Master Files, provided vital status information. The timeframe to determine survival was the date of diagnosis until censorship or the documented death date. We referred to the NCCN Clinical Practice Guidelines in Oncology (NCCN Guidelines) for Colon and Rectal Cancers to determine recommendations for the usual general treatment course by stage (eg, for colon cancer, surgery + chemotherapy recommended for stage III, radiation may be appropriate). ${ }^{20} \mathrm{We}$ described the number of patients in each comorbidity class who received the preferred treatment by stage (eg, surgery only for stage I colon cancer).

\section{Statistical Analysis}

We described continuous variables by reporting means and standard deviations, and used counts and frequencies for categorical variables. We described the burden of comorbid conditions by reporting counts of specific comorbidities and the weighted CCI score. Latent class analysis (LCA) was used to determine the number and composition of comorbidity-defined subgroups, or "classes," that fit the data most parsimoniously, using individual comorbidities in the model. ${ }^{21}$ LCA is a type of structural equation modeling used for finding subgroups of related cases from multivariate categorical data. The resulting latent classes represent groups of like individuals and distinguishes them from other individuals who are less alike. Using multiple iterations, the model estimates the posterior probability of class membership for each individual, and subsequently places the individual in the class for which the probability of membership is highest; results are reviewed and a final determination is made by the study team. In this study, LCA grouped comorbidities into the best fit number of classes based on their observed distribution in the population. ${ }^{22}$ We selected the most parsimonious number of classes by combining information from the Lo-Mendell-Rubin (LMR) test and Bayesian information criterion to triangulate the best fitting number of classes. We conducted a sensitivity analysis using LCA to examine comorbidity classes in patients diagnosed at $\leq 65$ years of age versus $\geq 66$ years of age. To describe general treatment approach by comorbidity class, we categorized the percent of patients by stage in each class who received different combinations of treatment. Bivariate and multivariable binomial regression models were used to estimate risk ratios with $95 \%$ confidence intervals, comparing each of the 3 comorbidity classes against a common referent class, class 1 . We categorized treatment regimen as either recommended or nonrecommended. To compare survival between comorbidity classes, we used Kaplan-Meier survival analysis and Cox proportional hazards regression. When performing survival analysis, we adjusted for multiple pairwise comparisons between comorbidity classes by using the Bonferroni correction. 
Hahn et al

\begin{tabular}{|c|c|}
\hline Variable & $\begin{array}{c}N(\%) \\
(\mathrm{N}=7,803)\end{array}$ \\
\hline \multicolumn{2}{|l|}{ Age at CRC diagnosis } \\
\hline Median (range), y & 66 y $(18-101 y)$ \\
\hline$\leq 40 \mathrm{y}$ & $239(3 \%)$ \\
\hline $41-64 y$ & $3,320(43 \%)$ \\
\hline $65-74 y$ & $2,071(27 \%)$ \\
\hline $75-84 y$ & $1,568(20 \%)$ \\
\hline$\geq 85 \mathrm{y}$ & $605(8 \%)$ \\
\hline \multicolumn{2}{|l|}{ Sex } \\
\hline Male & $4,029(52 \%)$ \\
\hline \multicolumn{2}{|l|}{ Race/Ethnicity } \\
\hline White & $4,046(52 \%)$ \\
\hline Black & $1,139(15 \%)$ \\
\hline Hispanic & $1,716(22 \%)$ \\
\hline Asian & $694(9 \%)$ \\
\hline Pacific Island & $78(1 \%)$ \\
\hline Native American/Alaskan & $13(<1 \%)$ \\
\hline Others & $34(<1 \%)$ \\
\hline Multiple & $63(1 \%)$ \\
\hline Unknown & $20(<1 \%)$ \\
\hline \multicolumn{2}{|l|}{ Body mass index } \\
\hline Missing & $81(1 \%)$ \\
\hline Underweight $\left(<18.5 \mathrm{~kg} / \mathrm{m}^{2}\right)$ & $167(2 \%)$ \\
\hline Normal weight $\left(18.5-24.9 \mathrm{~kg} / \mathrm{m}^{2}\right)$ & $2,352(30 \%)$ \\
\hline Overweight $\left(25.0-29.9 \mathrm{~kg} / \mathrm{m}^{2}\right)$ & $2,718(35 \%)$ \\
\hline Obese $\left(>30.0 \mathrm{~kg} / \mathrm{m}^{2}\right)$ & $2,485(32 \%)$ \\
\hline \multicolumn{2}{|l|}{ Smoking status } \\
\hline Ever & $3,348(43 \%)$ \\
\hline Never & $3,977(51 \%)$ \\
\hline Missing & $478(6 \%)$ \\
\hline \multicolumn{2}{|l|}{ Marital status } \\
\hline Unmarried & $2,754(35 \%)$ \\
\hline Married & $4,753(61 \%)$ \\
\hline Missing & $296(4 \%)$ \\
\hline \multicolumn{2}{|l|}{ Insurance type } \\
\hline Missing & $2(0 \%)$ \\
\hline Commercial & $3,674(47 \%)$ \\
\hline Medicaid & $68(1 \%)$ \\
\hline Medicare & $3,827(49 \%)$ \\
\hline Private pay & $232(3 \%)$ \\
\hline \multicolumn{2}{|l|}{ Years of KPSC membership } \\
\hline Mean (SD) & $17.2(13.28)$ \\
\hline Median & 14.3 \\
\hline Q1, Q3 & $6.1,26.4$ \\
\hline Range & $(0.0-60.0)$ \\
\hline \multicolumn{2}{|l|}{ Education $^{\mathrm{a}}$} \\
\hline High school or less & $3,244(44 \%)$ \\
\hline Some college or less & $2,320(32 \%)$ \\
\hline College or higher & $1,768(24 \%)$ \\
\hline
\end{tabular}

\begin{tabular}{|c|c|}
\hline Variable & $\begin{array}{c}N(\%) \\
(N=7,803)\end{array}$ \\
\hline \multicolumn{2}{|l|}{ Income $^{a}$} \\
\hline$<\$ 15,000$ & $718(10 \%)$ \\
\hline$\$ 15,000-\$ 34,999$ & $1,297(18 \%)$ \\
\hline$\$ 35,000-\$ 49,999$ & $996(14 \%)$ \\
\hline$\$ 50,000-\$ 74,999$ & $1,403(19 \%)$ \\
\hline$\$ 75,000-\$ 99,999$ & $1,021(14 \%)$ \\
\hline$\$ 100,000-\$ 149,999$ & $1,133(15 \%)$ \\
\hline$\geq \$ 150,000$ & $763(10 \%)$ \\
\hline \multicolumn{2}{|l|}{ Histology } \\
\hline Adenocarcinoma & $7,136(91 \%)$ \\
\hline Carcinoid & $252(3 \%)$ \\
\hline Signet ring & $105(1 \%)$ \\
\hline Other & $310(4 \%)$ \\
\hline \multicolumn{2}{|l|}{ Stage } \\
\hline $0-1$ & $3,294(42 \%)$ \\
\hline II & $1,690(22 \%)$ \\
\hline III & $1,724(22 \%)$ \\
\hline IV & $1,095(14 \%)$ \\
\hline \multicolumn{2}{|l|}{ Treatment received } \\
\hline Chemotherapy & $2,681(34 \%)$ \\
\hline Radiation therapy & $754(10 \%)$ \\
\hline Surgery & $6,924(89 \%)$ \\
\hline No surgery & $402(5 \%)$ \\
\hline \multicolumn{2}{|c|}{$\mathrm{CCl}$ (weighted), within 4 years prior to diagnosis } \\
\hline Mean (SD) & $1.8(2.32)$ \\
\hline 0 & $3,268(41 \%)$ \\
\hline 1 & $1,457(19 \%)$ \\
\hline 2 & $1,046(13 \%)$ \\
\hline 3 & $588(8 \%)$ \\
\hline$\geq 4$ & $1,444(19 \%)$ \\
\hline \multicolumn{2}{|l|}{$\mathrm{CCl}$, within 4 years prior to diagnosis } \\
\hline Myocardial infarction & $528(7 \%)$ \\
\hline Congestive heart failure & $625(8 \%)$ \\
\hline Peripheral vascular disease & $898(12 \%)$ \\
\hline Cerebrovascular disease & $604(8 \%)$ \\
\hline Dementia & $66(1 \%)$ \\
\hline Chronic pulmonary disease & $1,671(21 \%)$ \\
\hline $\begin{array}{l}\text { Connective tissue disease/rheumatic } \\
\text { disease }\end{array}$ & $194(2 \%)$ \\
\hline Peptic ulcer disease & $121(2 \%)$ \\
\hline Mild liver disease & $350(4 \%)$ \\
\hline Diabetes without complications & $888(11 \%)$ \\
\hline Diabetes with complications & $1,123(14 \%)$ \\
\hline Paraplegia \& hemiplegia & $58(1 \%)$ \\
\hline Renal disease & $1,361(17 \%)$ \\
\hline Cancer & $730(9 \%)$ \\
\hline Moderate or severe liver disease & $24(<1 \%)$ \\
\hline Metastatic carcinoma & $134(2 \%)$ \\
\hline AIDS/HIV & $21(<1 \%)$ \\
\hline
\end{tabular}

Abbreviations: $\mathrm{CCl}$, Charlson comorbidity index; CRC, colorectal cancer; KPSC, Kaiser Permanente Southern California.

${ }^{a}$ Counts are estimated by multiplying group totals by average percent.

\section{Results}

We identified 7,803 patients with CRC (Table 1). Median age at diagnosis was 66 years, and $46 \%$ were diagnosed at $\leq 65$ years of age; $52 \%$ were male, $22 \%$ were Hispanic, $15 \%$ were black, $10 \%$ were Asian/Pacific Islander, and $52 \%$ were white. The mean number of years for KPSC membership prior to CRC diagnosis was $17(\mathrm{SD}, 13)$, and the interquartile range was 6 years (Q1) to 26 years (Q3). Two-thirds of the pa- tients had a BMI $\geq 25 \mathrm{~kg} / \mathrm{m}^{2}$. The stage distribution was $42 \%$ stage $0-I, 22 \%$ stage II, $22 \%$ stage III, and $14 \%$ stage IV. Most patients (89\%) received surgery, $34 \%$ received chemotherapy, $10 \%$ received radiation therapy, and $5 \%$ received no treatment. The mean CCI score was 1.8 (SD, 2.3). Overall, approximately one-fifth of the patients (19\%) had a weighted CCI score of $\geq 4,40 \%$ had between 1 and 3 , and the remaining $41 \%$ had a score of zero. 


\section{Comorbidity Classes}

LCA resulted in 4 distinct classes by comorbidity types (LMR, $P<.001$; Table 2); detailed statistical results can be seen in supplemental eAppendix 1 (available with this article at JNCCN.org). Both the LMR test and the Bayesian information criterion agreed on the number of classes. Class 1 included relatively younger, healthy individuals with few comorbidities, representing $67 \%$ of the sample, with a median age at diagnosis of 62 years (Table 3 ). Although still relatively infrequent, the most common comorbidities in this class were chronic pulmonary disease $(15 \%)$ and diabetes without complications (13\%). Class 2, representing $15 \%$ of the total sample, was characterized mainly by patients with vascular and/or respiratory disease: $22 \%$ myocardial infarction, $27 \%$ congestive heart failure, $43 \%$ peripheral vascular disease, and $27 \%$ cerebrovascular disease. The median age at diagnosis for class 2 was 77 years, with a mean CCI of $3.3(\mathrm{SD}, 1.9)$. Other conditions in class 2 included renal disease $(32 \%)$ and uncomplicated diabetes (19\%). Class 3, representing $13 \%$ of the sample, was characterized mainly by the presence of diabetes with microvascular complications (78\%), with a high prevalence of accompanying renal disease $(60 \%)$ and a relatively low prevalence of respiratory or cardiovascular disease. The median age of class 3 was 76 years, with a mean CCI of 3.6 (SD, 1.8). Class 4 , representing $5 \%$ of the total sample, had the highest burden of comorbid conditions, with an average CCI of 7.2 (SD, 1.9). The median age of 71 years was slightly younger compared with classes 2 and 3. This class was dominated by patients with complicated diabetes $(83 \%)$, renal disease $(87 \%)$, congestive heart failure $(72 \%)$, peripheral vascular disease (70\%), chronic pulmonary disease $(54 \%)$, myocardial infarction (43\%), and cerebrovascular disease (38\%). Less common comorbid conditions in class 4 included cancer (other than the index CRC; 21\%) and liver disease $(8 \%)$. We found a higher percentage of eversmokers in classes 2 and 4 compared with classes 1 and 3 ( $54 \%$ vs $40 \%$, respectively; $P<.001$ ). The overlap by class of the most common comorbidities, including diabetes and vascular and respiratory diseases, is represented in Figure 2.

Results from the sensitivity analysis indicate little difference in comorbidity types and subsequent class membership assignment between patients diagnosed at $\leq 65$ years of age versus those diagnosed at $\geq 66$ years of age (supplemental eAppendix 2). The optimal

\begin{tabular}{|llll|}
\hline \multirow{2}{*}{ Table 2. Classes by Comorbidity Types } \\
\cline { 2 - 4 } & \multicolumn{3}{c|}{ Number of Classes } \\
\hline Bayesian information criterion & 55,490 & 4 & 5 \\
\hline LMR test & 2 vs 3 & 3 vs 4 & 4 vs 5 \\
LMR $P$ Value & $<.001$ & $<.001$ & .0895 \\
\hline
\end{tabular}

Choice of the most parsimonious number of classes was based on information from parsimony indices (Lo-Mendell-Rubin [LMR] test), and model comparison using the Bayesian Information Criterion to triangulate the best fitting number of classes.

number of classes was 4 , the same as the results for our total cohort, and the comorbidity patterns were similar, with class 1 representing those with the fewest comorbidities and class 4 with the most. Class 2 continued to be dominated by vascular/respiratory disease (although this was less frequent in patients diagnosed at $\leq 65$ years of age) and complicated diabetes with renal disease, and class 3 mainly by complicated diabetes.

\section{Treatment}

We described treatment receipt by class and stage (Table 4). There were statistically significant differences in treatment combinations by class within each stage group, broken out by stage 0-II (early stage), stage III, and stage IV. Table 5 shows an unadjusted and adjusted comparison of early- versus late-stage disease by class. Most patients with stage $0-$ II disease in each of the 4 classes received surgery only, in accordance with NCCN Guidelines recommendations. ${ }^{20}$ However, more class 1 members received multimodality treatment (surgery + chemotherapy or surgery + chemotherapy + radiation) for early-stage disease than those in the other 3 classes (although class 1 patients had a similar disease distribution of stage 0 , I, and II disease as the other classes). For patients with stage III disease, those in classes 1 and 3 more frequently received NCCN Guideline-recommended combined treatment (surgery + chemotherapy or surgery + chemotherapy + radiation) than those in classes 2 or 4. Those in classes 2 or 4 more frequently received only surgery for stage III disease $(40 \%$ and $46 \%$, respectively, vs $15 \%$ and $22 \%$ for classes 1 and 3 , respectively). This pattern was also observed among patients with stage IV disease, wherein members in classes 1 and 3 more frequently received more intensive surgery plus chemotherapy $(37 \%$ and $36 \%$, respectively, vs $17 \%$ and $17 \%$ for classes 2 and 4 , respectively). Patients in classes 2 and 4 with stage 
Hahn et al

\begin{tabular}{|c|c|c|c|c|c|}
\hline & $\begin{array}{c}\text { Class } 1 \\
(\mathrm{~N}=5,238)\end{array}$ & $\begin{array}{c}\text { Class 2 } \\
(\mathrm{N}=1,148)\end{array}$ & $\begin{array}{c}\text { Class } 3 \\
(\mathrm{~N}=998)\end{array}$ & $\begin{array}{c}\text { Class } 4 \\
(\mathrm{~N}=419)\end{array}$ & $P$ Value \\
\hline Patients in class & $67 \%$ & $15 \%$ & $13 \%$ & $5 \%$ & \\
\hline \multicolumn{6}{|l|}{ Age at CRC diagnosis } \\
\hline Median (range), y & $62(18-98)$ & $76(21-101)$ & $71(35-101)$ & $77(45-98)$ & $<.001$ \\
\hline$\leq 40 \mathrm{y}$ & $228(4 \%)$ & $5(0 \%)$ & $6(1 \%)$ & $0(0 \%)$ & \\
\hline $41-64 y$ & $2,798(53 \%)$ & $161(14 \%)$ & $314(31 \%)$ & $47(11 \%)$ & \\
\hline $65-74 y$ & $1,272(24 \%)$ & $344(30 \%)$ & $336(34 \%)$ & $119(28 \%)$ & \\
\hline $75-84 y$ & $732(14 \%)$ & $415(36 \%)$ & $250(25 \%)$ & $171(41 \%)$ & \\
\hline $285 \mathrm{y}$ & $208(4 \%)$ & $223(19 \%)$ & $92(9 \%)$ & $82(20 \%)$ & \\
\hline \multicolumn{6}{|l|}{ Years of enrollment } \\
\hline Mean (SD) & $15.2(12.6)$ & $21.7(13.5)$ & $19.7(13.5)$ & $24.6(13.8)$ & \\
\hline Median & 12.0 & 19.6 & 17.9 & 23.2 & \\
\hline Q1, Q3 & $4.6,23.2$ & $10.7,32.4$ & $8.3,29.7$ & $13.0,35.8$ & \\
\hline Range & $(0-58.5)$ & $(0-59.4)$ & $(0-59.9)$ & $(0.8-60.0)$ & \\
\hline \multicolumn{6}{|l|}{ Stage } \\
\hline $0-11$ & $64 \%$ & $66 \%$ & $62 \%$ & $68 \%$ & \\
\hline III-IV & $36 \%$ & $33 \%$ & $37 \%$ & $31 \%$ & \\
\hline Race/Ethnicity & & & & & $<.001$ \\
\hline White & $2,625(50 \%)$ & $739(64 \%)$ & $440(44 \%)$ & $242(58 \%)$ & \\
\hline Black & $689(13 \%)$ & $174(15 \%)$ & $206(21 \%)$ & $70(17 \%)$ & \\
\hline Hispanic & $1,243(24 \%)$ & $144(13 \%)$ & $249(25 \%)$ & $80(19 \%)$ & \\
\hline Asian & $530(10 \%)$ & $70(6 \%)$ & $72(7 \%)$ & $22(5 \%)$ & \\
\hline Pacific Island & $55(1 \%)$ & $6(1 \%)$ & $16(2 \%)$ & $1(<1 \%)$ & \\
\hline Native American/Alaskan & $10(<1 \%)$ & $1(<1 \%)$ & $2(<1 \%)$ & $0(0 \%)$ & \\
\hline Others & $25(<1 \%)$ & $5(<1 \%)$ & $3(<1 \%)$ & $1(<1 \%)$ & \\
\hline Multiple & $48(1 \%)$ & $6(1 \%)$ & $7(1 \%)$ & $2(<1 \%)$ & \\
\hline Unknown & $13(<1 \%)$ & $3(<1 \%)$ & $3(<1 \%)$ & $1(<1 \%)$ & \\
\hline Body mass index & & & & & $<.001$ \\
\hline Missing & $72(1 \%)$ & $5(<1 \%)$ & $3(<1 \%)$ & $1(<1 \%)$ & \\
\hline Underweight $\left(<18.5 \mathrm{~kg} / \mathrm{m}^{2}\right)$ & $108(2 \%)$ & $37(3 \%)$ & $13(1 \%)$ & $9(2 \%)$ & \\
\hline Normal weight $\left(18.5-24.9 \mathrm{~kg} / \mathrm{m}^{2}\right)$ & $1,570(30 \%)$ & $434(38 \%)$ & $244(24 \%)$ & $104(25 \%)$ & \\
\hline Overweight $\left(25.0-29.9 \mathrm{~kg} / \mathrm{m}^{2}\right)$ & $1,880(36 \%)$ & $364(32 \%)$ & $339(34 \%)$ & $135(32 \%)$ & \\
\hline Obese $\left(>30.0 \mathrm{~kg} / \mathrm{m}^{2}\right)$ & $1,608(31 \%)$ & $308(27 \%)$ & $399(40 \%)$ & $170(41 \%)$ & \\
\hline Smoking status & & & & & $<.001$ \\
\hline Ever & $2,034(39 \%)$ & $627(55 \%)$ & $460(46 \%)$ & $227(54 \%)$ & \\
\hline Never & $2,848(54 \%)$ & $471(51 \%)$ & $480(48 \%)$ & $178(42 \%)$ & \\
\hline Missing & $356(7 \%)$ & $50(4 \%)$ & $58(6 \%)$ & $14(3 \%)$ & \\
\hline
\end{tabular}

Abbreviations: $\mathrm{CCl}$, Charlson comorbidity index; CRC, colorectal cancer.

(continued on next page)

IV disease more frequently received no treatment (29\% and 37\%, respectively) compared with those in classes 1 and 3. Across all stages, patients in class 4 consistently received less aggressive treatment, followed by those in class 2. Although unadjusted analyses were not significant, when adjusting for patient race/ethnicity, marital status, sex, and age, we found that, compared with those in class 1 , those in classes 2 and 4 with advanced disease (stage III-IV) were significantly less likely to receive the recommended treatment course (class 2: odds ratio [OR], 0.45; 95\% CI, 0.28-0.74 and class 4: OR, 0.5; 95\% CI, 0.37-0.67).

\section{Survival by Stage and Comorbidity Class}

Kaplan-Meier estimates revealed a statistically significant association between comorbidity class membership and OS $(P<.001)$. Class 4 had the poorest $\mathrm{OS}$, with a median survival of 2.6 years
(Figure 3). In addition, survival appeared to be better for individuals in class 3 than for those in class 2 , but did not reach statistical significance after correcting for multiple comparisons $(P=.01$, Bonferroni corrected value). Unadjusted analyses of survival by comorbidity class, stratified by stage, showed a significant effect of comorbidity class for all stages (Figure 4). After adjustment for stage, age, sex, race/ethnicity, BMI, and marital status, comorbidity class was still associated with survival $(P<0.001$, Bonferroni corrected value). There were 6 total comparisons for the classes, setting the nominal $P$ value for statistical significance at $0.0083(0.05 / 6)$. Stage of disease $\left(\chi^{2}, 315\right)$ explained more variability in survival than comorbidity class $\left(\chi^{2}, 68\right)$. There was a statistically significant interaction between comorbidity class and stage (likelihood ratio test, $\mathrm{P}<.001)$. Comorbidity class was a slightly stronger 
Colorectal Cancer Comorbidity Profiles

\begin{tabular}{|c|c|c|c|c|c|}
\hline & $\begin{array}{c}\text { Class } 1 \\
(\mathrm{~N}=5,238)\end{array}$ & $\begin{array}{c}\text { Class } 2 \\
(\mathrm{~N}=1,148)\end{array}$ & $\begin{array}{c}\text { Class } 3 \\
(\mathrm{~N}=998)\end{array}$ & $\begin{array}{c}\text { Class } 4 \\
(\mathrm{~N}=419)\end{array}$ & $P$ Value \\
\hline $\mathrm{CCl}$ (weighted), within 4 years prior to diagnosis & & & & & $<.001$ \\
\hline Mean (SD) & $0.6(1.25)$ & $3.3(1.89)$ & $3.6(1.76)$ & $7.2(1.96)$ & \\
\hline 0 & $3,268(62 \%)$ & $0(0 \%)$ & $0(0 \%)$ & $0(0 \%)$ & \\
\hline 1 & $1,320(25 \%)$ & $134(12 \%)$ & $3(<1 \%)$ & $0(0 \%)$ & \\
\hline 2 & $397(8 \%)$ & $290(25 \%)$ & $359(36 \%)$ & $0(0 \%)$ & \\
\hline 3 & $147(3 \%)$ & $305(27 \%)$ & $135(14 \%)$ & $1(<1 \%)$ & \\
\hline$\geq 4$ & $106(2 \%)$ & $419(36 \%)$ & $501(50 \%)$ & $418(100 \%)$ & \\
\hline \multicolumn{6}{|l|}{$\mathrm{CCl}$, within 4 years prior to diagnosis } \\
\hline Myocardial infarction & $57(1 \%)$ & $251(22 \%)$ & $39(4 \%)$ & $181(43 \%)$ & $<.001$ \\
\hline Congestive heart failure & $0(0 \%)$ & $313(27 \%)$ & $10(1 \%)$ & $302(72 \%)$ & $<.001$ \\
\hline Peripheral vascular disease & $0(0 \%)$ & $492(43 \%)$ & $112(11 \%)$ & $294(70 \%)$ & $<.001$ \\
\hline Cerebrovascular disease & $81(2 \%)$ & $311(27 \%)$ & $54(5 \%)$ & $158(38 \%)$ & $<.001$ \\
\hline Dementia & $0(0 \%)$ & $44(4 \%)$ & $0(0 \%)$ & $22(5 \%)$ & $<.001$ \\
\hline Chronic pulmonary disease & $776(15 \%)$ & $513(45 \%)$ & $154(15 \%)$ & $228(54 \%)$ & $<.001$ \\
\hline Connective tissue disease/rheumatic disease & $85(2 \%)$ & $61(5 \%)$ & $27(3 \%)$ & $21(5 \%)$ & $<.001$ \\
\hline Peptic ulcer disease & $34(1 \%)$ & $49(4 \%)$ & $20(2 \%)$ & $18(4 \%)$ & $<.001$ \\
\hline Mild liver disease & $203(4 \%)$ & $65(6 \%)$ & $50(5 \%)$ & $32(8 \%)$ & $<.001$ \\
\hline Diabetes without complications & $665(13 \%)$ & $223(19 \%)$ & $0(0 \%)$ & $0(0 \%)$ & $<.001$ \\
\hline Diabetes with complications & $0(0 \%)$ & $0(0 \%)$ & $774(78 \%)$ & $349(83 \%)$ & $<.001$ \\
\hline Paraplegia and hemiplegia & $0(0 \%)$ & $38(3 \%)$ & $5(1 \%)$ & $15(4 \%)$ & $<.001$ \\
\hline Renal disease & $29(1 \%)$ & $371(32 \%)$ & $595(60 \%)$ & $366(87 \%)$ & $<.001$ \\
\hline Cancer & $354(7 \%)$ & $170(15 \%)$ & $116(12 \%)$ & $90(21 \%)$ & $<.001$ \\
\hline Moderate or severe liver disease & $6(<1 \%)$ & $11(1 \%)$ & $2(<1 \%)$ & $5(1 \%)$ & $<.001$ \\
\hline Metastatic carcinoma & $69(1 \%)$ & $33(3 \%)$ & $21(2 \%)$ & $11(3 \%)$ & .001 \\
\hline AIDS/HIV & $16(<1 \%)$ & $4(<1 \%)$ & $0(0 \%)$ & $1(<1 \%)$ & .357 \\
\hline Number of Charlson comorbidities, within 4 years prior to diagnosis & $0.5(0.68)$ & $2.6(1.08)$ & $2.8(1.17)$ & $5.8(1.21)$ & $<.001$ \\
\hline 0 & $3,268(62 \%)$ & $0(0 \%)$ & $0(0 \%)$ & $0(0 \%)$ & \\
\hline 1 & $1,560(30 \%)$ & $139(12 \%)$ & $171(17 \%)$ & $0(0 \%)$ & \\
\hline 2 & $353(7 \%)$ & $456(40 \%)$ & $238(24 \%)$ & $0(0 \%)$ & \\
\hline 3 & $48(1 \%)$ & $366(32 \%)$ & $314(31 \%)$ & $7(2 \%)$ & \\
\hline 4 & $9(<1 \%)$ & $122(11 \%)$ & $213(21 \%)$ & $37(9 \%)$ & \\
\hline 5 & $0(0 \%)$ & $44(4 \%)$ & $54(5 \%)$ & $129(31 \%)$ & \\
\hline 6 & $0(0 \%)$ & $17(1 \%)$ & $7(1 \%)$ & $129(31 \%)$ & \\
\hline$\geq 7$ & $0(0 \%)$ & $4(<1 \%)$ & $1(<1 \%)$ & $117(28 \%)$ & \\
\hline
\end{tabular}

Abbreviations: $\mathrm{CCl}$, Charlson comorbidity index; CRC, colorectal cancer.

source of variability in survival than the CCI score alone (log-rank $\chi^{2}, 413$ vs 380 , respectively).

Additionally, we examined hazard ratios (HRs) for unadjusted and adjusted models to compare survival by class, as well as the stage-specific HRs for an adjusted model, which included a class-by-stage interaction (Table 6). All classes had worse survival than class 1 , with class 4 having the poorest survival compared with all other classes, both before and after covariate adjustment. Furthermore, the differences in survival between classes were greatest among patients who had early-stage disease (stage $0-$ II) and were not statistically significant for those with stages III-IV, except when comparing class 4 with the others.

\section{Discussion}

In this population-based study from a large integrated healthcare system, we found that patients with CRC could be grouped into 4 distinct classes defined by clusters of comorbid conditions, and that the resulting comorbidity classes were associated with significant differences in both receipt of stage-specific treatment and survival. In our primary analysis, comorbidity classes 1 through 4 were defined by progressively greater mean CCI scores and by recognizable patterns of cardiovascular, respiratory, and metabolic disorders. Class 1 comprised most of the sample, and most patients in this class had no Charlson-defined comorbidities or only one comorbid condition. Close to half of our cohort (46\%) was diagnosed with CRC at $\leq 65$ years of age, with a median age at diagnosis of 66 years. This sample is somewhat younger than those reported in other CRC cohort studies, and our resulting comorbidity profiles may be influenced by the age distribution (eg, younger patients are less likely to have multimorbidity). Patients in class 1 more frequently received NCCN Guideline-recommended stage-specific CRC treatment and had better survival than those in any of the other classes, despite having a similar stage distribution as those in 
Hahn et al

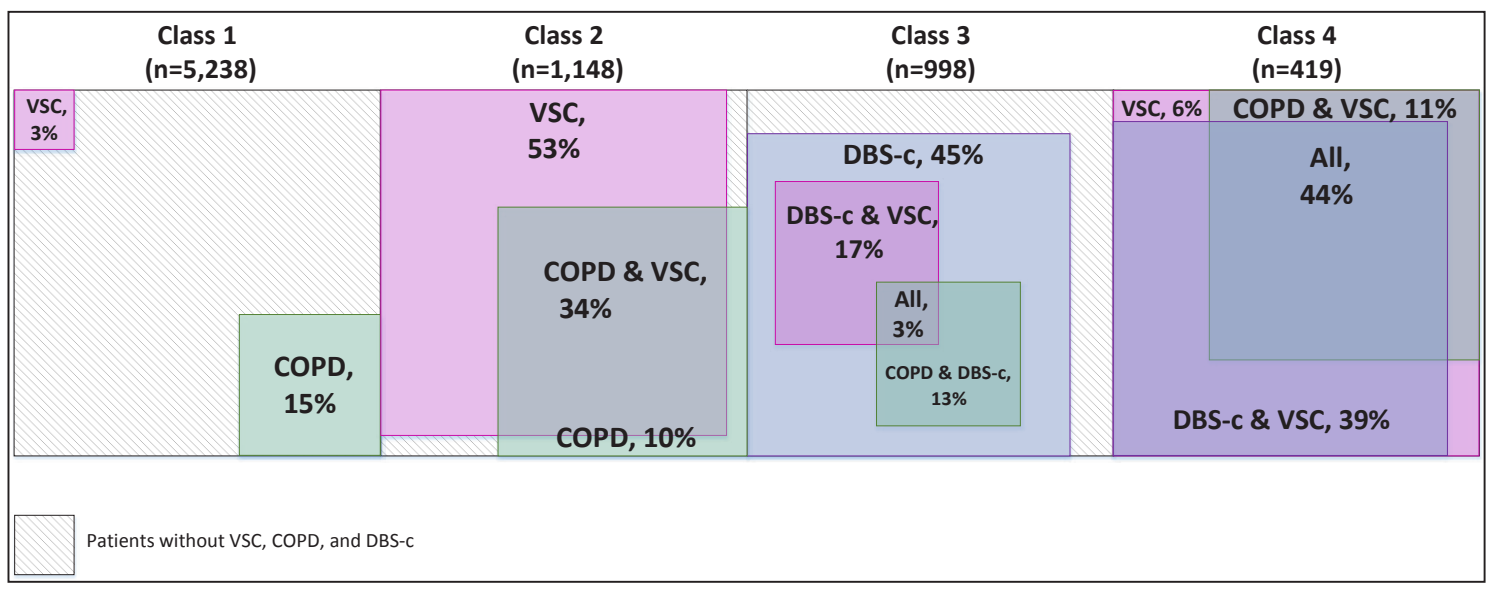

Figure 2. Overlap of the most common Charlson-defined comorbidities by latent class analysis-defined classes ( $\mathrm{N}=7,803)$.

Abbreviations: CCl, Charlson comorbidity index; COPD, chronic obstructive pulmonary disease; DBS-c, complicated diabetes; VSC, vascular disease.

the other 3 classes. Additionally, patients in class 1 were more frequently treated aggressively with multimodality therapy. In contrast, class 4 included only $5 \%$ of the sample and was characterized by the presence of multiple $(\geq 4)$ comorbid conditions, a relatively high frequency of not receiving recommended stage-specific treatment or receiving no treatment, and markedly worse OS than class 1 . Choice of less aggressive treatment for those in class 4 may be clinically appropriate, given the high burden of comorbidity in this class. Other studies have found an impact of single comorbidities on treatment selection in patients with CRC (eg, heart failure, chronic obstructive pulmonary disease $)^{23,24}$; our findings add to the existing body of knowledge by describing how these comorbidities co-occur, and the subsequent influence on treatment selection and OS.

Classes 2 and 3 appeared very similar in terms of median age at diagnosis and mean CCI score, and represented a similar proportion of the larger cohort. However, patients in class 2, which was characterized primarily by the presence of cardiovascular or cardiorespiratory diseases, were less likely to receive recommended stage-specific treatment than those in class 3, which was characterized primarily by diabetes with microvascular complications, including kidney disease. Patients in class 3 were less likely to receive no treatment than those in class 2 across all disease

\section{Table 4. Initial Treatment Received by Comorbidity Class, Stratified by Stage}

\begin{tabular}{|c|c|c|c|c|c|}
\hline & Class 1 & Class 2 & Class 3 & Class 4 & $P$ Value \\
\hline Treatment combination for stage $0-I I$ & $N=3,307$ & $N=764$ & $N=625$ & $N=\mathbf{2 8 8}$ & $<.001$ \\
\hline None & $3 \%$ & $6 \%$ & $5 \%$ & $7 \%$ & \\
\hline Surgery only (usual) & $81 \%$ & $85 \%$ & $83 \%$ & $85 \%$ & \\
\hline Surgery + chemotherapy & $7 \%$ & $3 \%$ & $5 \%$ & $2 \%$ & \\
\hline Surgery + radiation & $<1 \%$ & $1 \%$ & $<1 \%$ & $1 \%$ & \\
\hline Surgery + chemotherapy + radiation & $6 \%$ & $4 \%$ & $4 \%$ & $3 \%$ & \\
\hline Treatment combination for stage III & $N=1,178$ & $N=230$ & $N=231$ & $N=85$ & $<.001$ \\
\hline None & $1 \%$ & $<1 \%$ & $0 \%$ & $1 \%$ & \\
\hline Surgery only & $15 \%$ & $40 \%$ & $22 \%$ & $46 \%$ & \\
\hline Surgery + chemotherapy (usual) & $63 \%$ & $48 \%$ & $62 \%$ & $36 \%$ & \\
\hline Surgery + radiation & $1 \%$ & $<1 \%$ & $<1 \%$ & $1 \%$ & \\
\hline Surgery + chemotherapy + radiation (usual) & $16 \%$ & $8 \%$ & $13 \%$ & $9 \%$ & \\
\hline Treatment combination for stage IV & $N=753$ & $N=154$ & $N=142$ & $N=46$ & $<.001$ \\
\hline None & $14 \%$ & $29 \%$ & $21 \%$ & $37 \%$ & \\
\hline Surgery only & $13 \%$ & $21 \%$ & $13 \%$ & $28 \%$ & \\
\hline Surgery + chemotherapy & $37 \%$ & $17 \%$ & $36 \%$ & $17 \%$ & \\
\hline Surgery + radiation & $1 \%$ & $1 \%$ & $0 \%$ & $0 \%$ & \\
\hline Surgery + chemotherapy + radiation & $3 \%$ & $0 \%$ & $1 \%$ & $0 \%$ & \\
\hline
\end{tabular}


Colorectal Cancer Comorbidity Profiles

\begin{tabular}{|c|c|c|c|c|}
\hline Comparison $^{\mathrm{a}}$ & $\begin{array}{c}\text { Unadjusted OR } \\
(95 \% \mathrm{Cl})\end{array}$ & $\begin{array}{l}\text { Adjusted OR } \\
(95 \% \mathrm{Cl})\end{array}$ & $\begin{array}{l}\text { Within Stage 0-II } \\
\quad(95 \% \mathrm{CI})\end{array}$ & $\begin{array}{c}\text { Within Stage III-IV } \\
(95 \% \mathrm{Cl})\end{array}$ \\
\hline Class 2 vs 1 & $0.83(0.62-1.11)$ & $0.77(0.58-1.03)$ & $1.42(0.76-2.65)$ & $0.45(0.28-0.74)$ \\
\hline Class 3 vs 1 & $0.99(0.8-1.23)$ & $1.02(0.82-1.28)$ & $1.16(0.77-1.74)$ & $0.93(0.67-1.29)$ \\
\hline Class 4 vs 1 & $0.82(0.68-0.99)$ & $0.78(0.64-0.95)$ & $1.36(0.91-2.03)$ & $0.5(0.37-0.68)$ \\
\hline Class 2 vs 3 & $0.84(0.6-1.18)$ & $0.76(0.54-1.05)$ & $1.22(0.6-2.49)$ & $0.49(0.28-0.85)$ \\
\hline Class 2 vs 4 & $1.02(0.73-1.41)$ & $0.99(0.73-1.35)$ & $1.04(0.51-2.11)$ & $0.9(0.53-1.54)$ \\
\hline Class 3 vs 4 & $1.21(0.93-1.57)$ & $1.31(1.01-1.70)$ & $0.85(0.5-1.44)$ & $1.85(1.24-2.77)$ \\
\hline Class main effect $P$ value & .025 & .001 & .038 & \\
\hline Stage main effect $P$ value & NA & $<.001$ & $<.001$ & \\
\hline Class-by-Stage interaction $P$ value & NA & NA & $<.001$ & \\
\hline
\end{tabular}

Abbreviations: NA, not applicable; ORs, odds ratios.

${ }^{a}$ All comparisons adjusted for multiple comparisons; multivariate models adjusted for patient stage, race/ethnicity, marital status, sex, and age.

stages and had slightly better OS, although the latter difference was not statistically significant. This suggests that complicated diabetes (eg, represented by those in class 3 ) was less likely to influence treatment selection than underlying cardiovascular or respiratory disease. Although our study did not discriminate between type 1 and type 2 diabetes, other studies have found that type 2 is associated with a higher risk of developing $\mathrm{CRC}$, and that among patients diagnosed with $\mathrm{CRC}$, those with diabetes are more likely to die. ${ }^{14,25}$ It is possible that the less intensive treatment for patients in class 2 could be explained by treatment contraindications for those with clinically significant cardiovascular disease at the time of CRC diagnosis, or patient refusal of more intensive therapy.

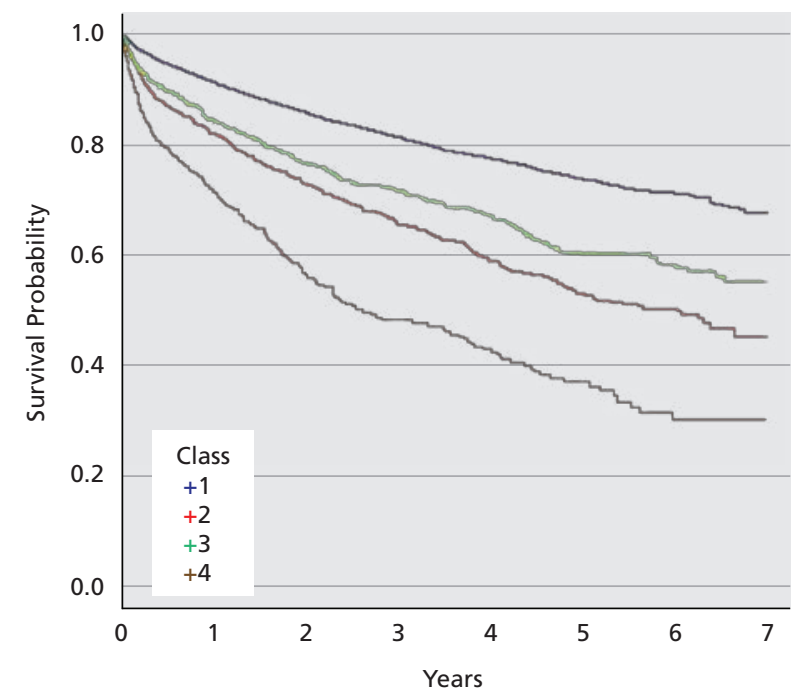

Figure 3. Kaplan-Meier survival trends by class profile for patients with first time diagnoses of colorectal cancer $(\mathrm{N}=7,803)$.
Although comorbidity class alone may not explain treatment selection (eg, although age alone should not be a contraindication, age at diagnosis can play a significant role in treatment selection ${ }^{26}$ ), our findings suggest that clusters of comorbidities do have a significant association with treatment. This is in line with other studies that show that patients with CRC with multiple comorbidities are less likely to receive chemotherapy. ${ }^{8,27}$ Additionally, we found a high prevalence of smoking-related disease (respiratory and vascular) in classes 2 and 4 .

Our study has several important implications for research and clinical practice. Although comorbid conditions are common in patients with CRC, the most common presentation we found was single or no comorbidity. In addition, although the high prevalence of respiratory and cardiac conditions in our sample is not surprising, we found a common comorbidity profile dominated by diabetes with renal complications. These comorbidity profiles appear to be associated with treatment decision-making. It is noteworthy that the effect of comorbidity class on survival persisted when we adjusted for stage. In addition, there was a statistically significant interaction between comorbidity class and stage, and LCA was found to be superior to using the CCI alone. We reported similar findings in a large cohort of patients with lung cancer. ${ }^{28}$ More fundamentally, our findings represent a shift away from simple counting of comorbid conditions and toward a richer, more nuanced understanding of how comorbidities cluster within groups of patients with CRC. We hope that this approach will lead to potentially actionable findings for clinical settings. Our findings indicate the need to learn more about the effect of comorbidity 
A

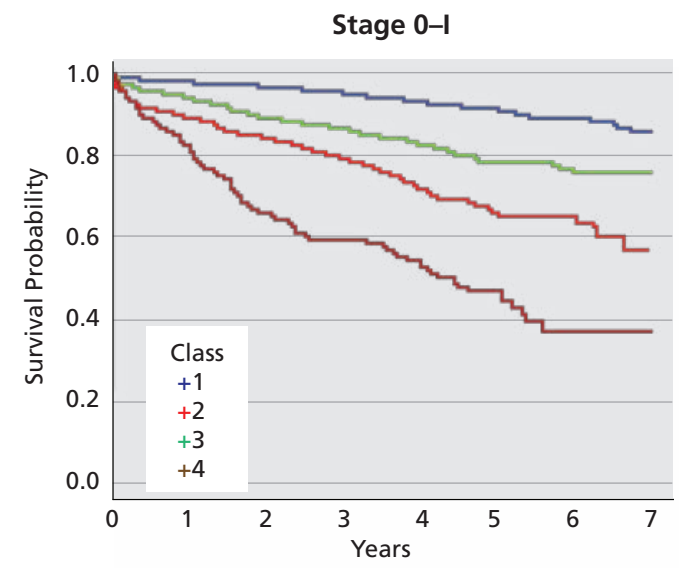

C

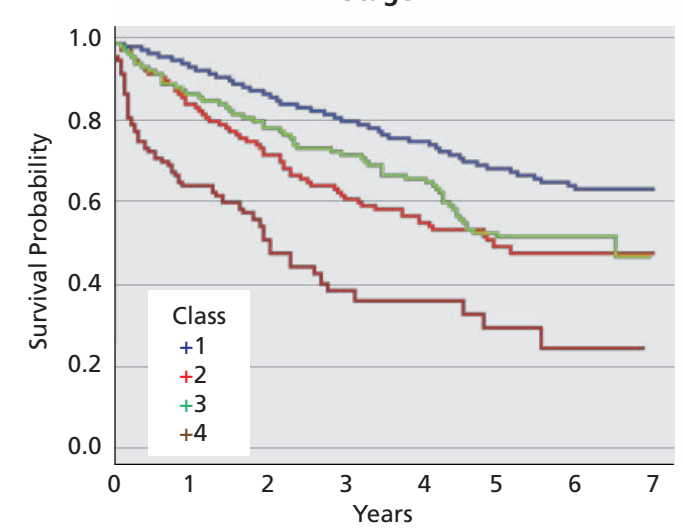

B

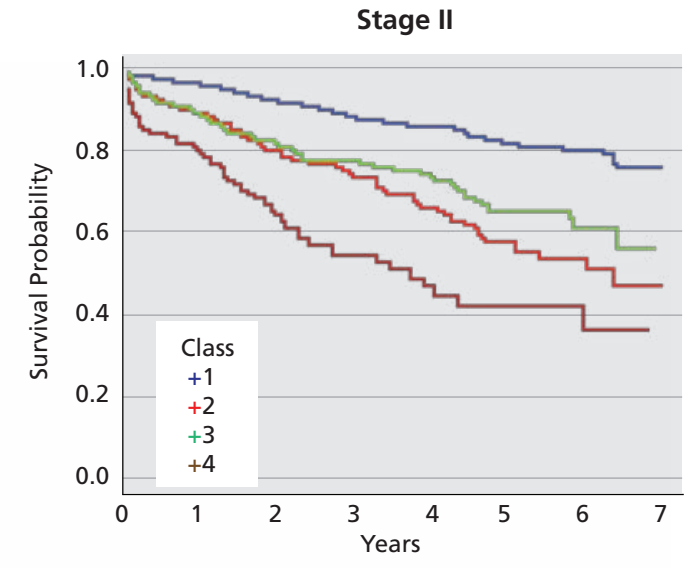

D

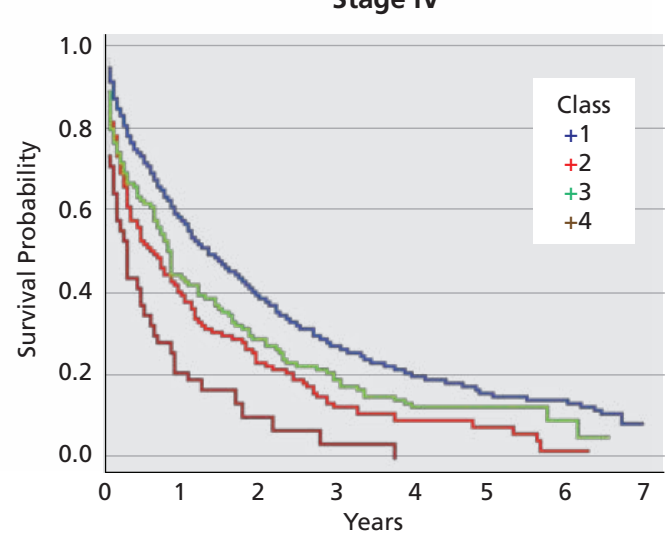

Figure 4. Kaplan-Meir survival trends by class profile, stratified by cancer stage, for patients with first time diagnoses of colorectal cancer ( $\mathrm{N}=7,803$ ) (A) Stage 0-I, (B) stage II, (C) stage III, and (D) stage IV.

clusters on treatment tolerance and response, which will contribute to future recommendations for personalized treatment of CRC. It may be useful to have a common language to describe comorbidity clusters, enhancing future research efforts and potentially leading to a taxonomy to describe and evaluate comorbidities in cancer and other disease populations.

Important strengths of the study include the large sample, the community-based, integrated healthcare setting, and the availability of comprehensive EHRs with longitudinal data to capture the full burden of comorbid conditions. Additionally, we had the opportunity to include younger patients (eg, age 18-64 years) who are typically underrepresented in CRC research. Our results indicate that most younger patients have few comorbidities, as expected.

Limitations include the retrospective study design, lack of detailed treatment data (eg, adjuvant vs

\begin{tabular}{|c|c|c|c|c|c|c|}
\hline Comparison & $\begin{array}{l}\text { Unadjusted HR } \\
(95 \% \mathrm{Cl})\end{array}$ & $\begin{array}{l}\text { Adjusted HR } \\
(95 \% \mathrm{Cl})\end{array}$ & $\begin{array}{l}\text { Within Stage 0-I } \\
\quad(95 \% \mathrm{Cl})\end{array}$ & $\begin{array}{l}\text { Within Stage II } \\
(95 \% \mathrm{CI})\end{array}$ & $\begin{array}{l}\text { Within Stage III } \\
(95 \% \mathrm{Cl})\end{array}$ & $\begin{array}{l}\text { Within Stage IV } \\
\qquad(95 \% \mathrm{Cl})\end{array}$ \\
\hline Class 2 vs 1 & $2.06(1.77,2.39)$ & $1.67(1.42,1.95)$ & $3.34(2.16,5.18)$ & $2.04(1.26,3.3)$ & $1.36(0.88,2.1)$ & $1.17(0.82,1.67)$ \\
\hline Class 3 vs 1 & $1.63(1.38,1.92)$ & $1.51(1.27,1.8)$ & $2.29(1.36,3.87)$ & $2.02(1.17,3.46)$ & $1.4(0.89,2.21)$ & $1.19(0.82,1.72)$ \\
\hline Class 4 vs 1 & $3.47(2.86,4.21)$ & $3.54(2.88,4.35)$ & $7.34(4.51,11.94)$ & $3.83(2.1,6.98)$ & $2.86(1.65,4.96)$ & $2.13(1.19,3.81)$ \\
\hline Class 2 vs 3 & $1.27(1.04,1.54)$ & $1.1(0.9,1.34)$ & $1.46(0.85,2.5)$ & $1.01(0.56,1.82)$ & $0.97(0.56,1.68)$ & $0.98(0.62,1.55)$ \\
\hline Class 2 vs 4 & $0.59(0.48,0.74)$ & $0.47(0.38,0.59)$ & $0.46(0.28,0.75)$ & $0.53(0.28,1.01)$ & $0.48(0.26,0.89)$ & $0.55(0.29,1.03)$ \\
\hline Class 3 vs 4 & $0.47(0.37,0.59)$ & $0.43(0.34,0.54)$ & $0.31(0.18,0.56)$ & $0.53(0.27,1.04)$ & $0.49(0.26,0.93)$ & $0.56(0.29,1.06)$ \\
\hline
\end{tabular}

Abbreviation: HR, hazard ratio. 
neoadjuvant therapy), lack of cancer-specific mortality data, and the need to rely on retrospective collection of diagnostic codes in EHRs. It is possible that certain comorbid conditions would be more or less likely to be coded correctly in the records, leading to possible ascertainment bias. However, the DaCCI has been used to adjust for comorbidity in countless retrospective studies that relied on either administrative claims or EHRs, and has consistently been shown to correlate negatively with survival and other outcomes of interest. Future studies are needed to compare the advantages and disadvantages of these alternative approaches. A limitation of most comorbidity indices is that the presence or number of comorbidities does not necessarily reflect the severity of any given comorbid condition; therefore, future work in this area should include assessment of severity via laboratory and other results that could feasibly be captured from EHRs. Comorbid conditions also do not capture all possible clinical factors that may influence decision-making regarding cancer treatment planning, such as malnutrition or functional status. Additionally, our approach to the LCAs, which assigned class membership based on highest probability, may not be the optimal approach when many members have assignment probabilities of less than 0.5 ; however, it is worth noting that in our sample only $5 \%$ of members fell into this category (supplemental eAppendix 3). Finally, our survival analysis did not incorporate the classification uncertainty. Given that this would tend to underestimate class-covariate associations, regardless of how class membership is assigned, ${ }^{29}$ the differences we detected in survival patterns should have only been more dramatic than we found had we incorporated such classification error.

\section{Conclusions}

We identified 4 clinically distinct classes of comorbid conditions in patients with CRC. We found that these classes were associated with both treatment and OS, even when controlling for disease stage. $\mathrm{Pa}$ tients with multimorbidities with a high prevalence of cardiovascular disease were less likely to receive recommended treatment and had worse OS. Ultimately, this information can be used to provide personalized care for patients with CRC throughout the cancer care continuum.

\section{References}

1. Miller KD, Siegel RL, Lin CC, et al. Cancer treatment and survivorship statistics, 2016. CA Cancer J Clin 2016;66:271-289.

2. Parry $C$, Kent EE, Mariotto $A B$, et al. Cancer survivors: a booming population. Cancer Epidemiol Biomarkers Prev 2011;20:1996-2005.

3. Edwards BK, Brown ML, Wingo PA, et al. Annual report to the nation on the status of cancer, 1975-2002, featuring population-based trends in cancer treatment. J Natl Cancer Inst 2005;97:1407-1427.

4. Yoon SJ, Kim EJ, Seo HJ, Oh IH. The association between Charlson comorbidity index and the medical care cost of cancer: a retrospective study. Biomed Res Int 2015;2015:259341.

5. Janssen-Heijnen ML, Houterman S, Lemmens VE, et al. Prognostic impact of increasing age and co-morbidity in cancer patients: a population-based approach. Crit Rev Oncol Hematol 2005;55:231-240.

6. Keating NL, Landrum MB, Klabunde CN, et al. Adjuvant chemotherapy for stage III colon cancer: do physicians agree about the importance of patient age and comorbidity? J Clin Oncol 2008;26:2532-2537.

7. Søgaard M, Thomsen RW, Bossen KS, et al. The impact of comorbidity on cancer survival: a review. Clin Epidemiol 2013;5(Suppl 1):3-29.

8. Sarfati D, Tan L, Blakely T, Pearce N. Comorbidity among patients with colon cancer in New Zealand. N Z Med J 2011;124:76-88.

9. Shack LG, Rachet B, Williams EM, et al. Does the timing of comorbidity affect colorectal cancer survival? A population based study. Postgrad Med J 2010;86:73-78.

10. Lemmens VE, van Halteren AH, Janssen-Heijnen ML, et al. Adjuvant treatment for elderly patients with stage III colon cancer in the southern Netherlands is affected by socioeconomic status, gender, and comorbidity. Ann Oncol 2005;16:767-772.

11. Yancik R, Wesley MN, Ries LA, et al. Effect of age and comorbidity in postmenopausal breast cancer patients aged 55 years and older. JAMA 2001;285:885-892.
12. Zeber JE, Copeland LA, Hosek BJ, et al. Cancer rates, medical comorbidities, and treatment modalities in the oldest patients. Crit Rev Oncol Hematol 2008;67:237-242.

13. Meyerhardt JA, Catalano PJ, Haller DG, et al. Impact of diabetes mellitus on outcomes in patients with colon cancer. J Clin Oncol 2003;21:433-440.

14. Stein KB, Snyder CF, Barone BB, et al. Colorectal cancer outcomes, recurrence, and complications in persons with and without diabetes mellitus: a systematic review and meta-analysis. Dig Dis Sci 2010;55:1839_ 1851.

15. Ouellette JR, Small DG, Termuhlen PM. Evaluation of Charlson-age comorbidity index as predictor of morbidity and mortality in patients with colorectal carcinoma. J Gastrointest Surg 2004;8:1061-1067.

16. Koebnick C, Langer-Gould AM, Gould MK, et al. Sociodemographic characteristics of members of a large, integrated health care system: comparison with US Census Bureau data. Perm J 2012;16:37-41.

17. Charlson ME, Pompei P, Ales KL, MacKenzie CR. A new method of classifying prognostic comorbidity in longitudinal studies: development and validation. J Chronic Dis 1987;40:373-383.

18. de Groot V, Beckerman H, Lankhorst GJ, Bouter LM. How to measure comorbidity: a critical review of available methods. J Clin Epidemiol 2003;56:221-229.

19. Deyo RA, Cherkin DC, Ciol MA. Adapting a clinical comorbidity index for use with ICD-9-CM administrative databases. J Clin Epidemiol 1992;45:613-619.

20. Benson AB III, Venook AP, Cederquist L, et al. NCCN Clinical Practice Guidelines in Oncology: Colon Cancer. Version 2.2017. Accessed November 30, 2017. To view the most recent version of these guidelines, visit NCCN.org.

21. Muthen $B$, Muthen LK. Integrating person-centered and variable-centered analyses: growth mixture modeling with latent trajectory classes. Alcohol Clin Exp Res 2000;24:882-891. 
22. Huh J, Riggs NR, Spruijt-Metz D, et al. Identifying patterns of eating and physical activity in children: a latent class analysis of obesity risk. Obesity (Silver Spring) 2011;19:652-658.

23. Gross CP, McAvay GJ, Guo Z, Tinetti ME. The impact of chronic illnesses on the use and effectiveness of adjuvant chemotherapy for colon cancer. Cancer 2007;109:2410-2419.

24. Lemmens VE, Janssen-Heijnen ML, Verheij CD, et al. Co-morbidity leads to altered treatment and worse survival of elderly patients with colorectal cancer. Br J Surg 2005;92:615-623.

25. De Bruijn KM, Arends LR, Hansen BE, et al. Systematic review and metaanalysis of the association between diabetes mellitus and incidence and mortality in breast and colorectal cancer. Br J Surg 2013;100:1421-1429.
26. Quipourt V, Jooste V, Cottet V, et al. Comorbidities alone do not explain the undertreatment of colorectal cancer in older adults: a French population-based study. J Am Geriatr Soc 2011;59:694-698.

27. Sarfati D, Hill S, Blakely T, et al. The effect of comorbidity on the use of adjuvant chemotherapy and survival from colon cancer: a retrospective cohort study. BMC Cancer 2009;9:116.

28. Gould M, Munoz-Plaza C, Hahn E, et al. Comorbidity profiles and their effect on treatment selection and survival among patients with lung cancer. Ann Am Thorac Soc 2017;14:1571-1580.

29. Vermunt JK. Latent class modeling with covariates: two improved three step approaches. Polit Anal 2017;18:450-469.

\section{See JNCCN.org for supplemental online content.}

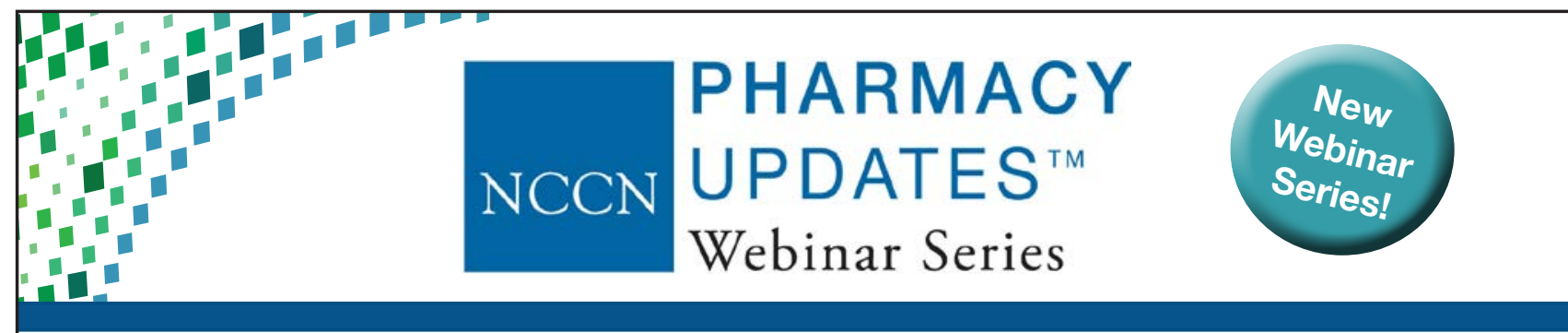

The availability of new therapeutic options and emergence of novel therapies continues to dramatically impact the oncology landscape. The NCCN Pharmacy Updates ${ }^{\mathrm{TM}}$ : Webinar Series is designed for pharmacists, nurses, and other members of the oncology care team to review the clinical and patient considerations that influence how to effectively optimize management of patients with various cancer types. The following webinars are currently scheduled:

- Immunotherapies in Metastatic Bladder Cancer Wednesday, January 31, 2018 · 5:30 - 6:30 PM ET

- Introduction to the NCCN Guidelines ${ }^{\circledR}$ for Myeloproliferative Neoplasms and a Comprehensive Review of the Use of JAK2 Inhibitors Friday, February 23, $2018 \cdot 4: 30$ - 5:30 PM ET

- Integrating New Treatment Options for Multiple Myeloma: Focus on Oral Therapies Wednesday, March 28, $2018 \cdot 5: 00$ - 6:00 PM ET

Visit education.nccn.org/pharmacy-updates to view topics and dates.

This program has been approved for AMA PRA Category 1 Credit TM for physicians and will award contact hours for nurses, pharmacists, and other health care professionals. Complete accreditation information is available online.

Supported by and educational funding provided by Amgen. Supported by an independent educational grant from Incyte. Supported by educational grants from: AstraZeneca; Celgene Corporation; Lilly: Novartis Pharmaceuticals Corporation; Pharmacyclics LLC, an AbbVie Company and Janssen Biotech, Inc., administered by Janssen Scientific Affairs, LLC; Prometheus Laboratories Inc; and Takeda Oncology. 